\title{
Quantifying the azimuthal plasmaspheric density structure and dynamics inferred from IMAGE EUV
}

\author{
Patrick Sibanda, ${ }^{1,2}$ Mark B. Moldwin, ${ }^{1}$ David A. Galvan, ${ }^{3}$ Bill R. Sandel, ${ }^{4}$ \\ and Terry Forrester ${ }^{4}$ \\ Received 11 January 2012; revised 29 September 2012; accepted 2 October 2012; published 8 November 2012.
}

[1] The extreme ultraviolet (EUV) imager on the IMAGE satellite provided the first global images of the plasmasphere leading to enhanced understanding of plasmapause structure and dynamics. However, few studies have investigated the structure and dynamics of the inner plasmasphere (regions interior to the plasmapause), which previous in situ observations have shown to often be highly structured. This study is the first to systematically analyze global images of the density structure of the inner plasmasphere by using data from the EUV imager on the IMAGE satellite. We find that the inner plasmasphere exhibits both fine and meso-scale structure characterized by rapid density fluctuations and density enhancements of varying amplitudes (factors of $\sim 2-5$ ) and spatial scales (from $10 \mathrm{~s}$ of minutes to 6 hours MLT) that occur regularly in the aftermath of geomagnetic storms. The level of variability within the azimuthal structure was found to increase with increasing geomagnetic activity. The observations suggest that some meso-scale azimuthal density structure observed in the inner plasmasphere is from "fossil" plasmapause features entrained inside the expanding and refilling plasmasphere.

Citation: Sibanda, P., M. B. Moldwin, D. A. Galvan, B. R. Sandel, and T. Forrester (2012), Quantifying the azimuthal plasmaspheric density structure and dynamics inferred from IMAGE EUV, J. Geophys. Res., 117, A11204, doi:10.1029/2012JA017522.

\section{Introduction}

[2] Over the years much has been learned about the structure and dynamics of the plasmasphere from ground-based, in situ and remote space-based sensing technologies. Various studies have provided a better understanding of many aspects of the plasmasphere such as the changes in plasmaspheric morphology in response to magnetic disturbances (i.e., plasmaspheric erosion and refilling processes) and the interplay among global magnetospheric convection and small scale electric fields [Carpenter et al., 2000]. Several studies have shown the plasmaspheric density structure to be highly spatially variable in response to changes in geomagnetic activity [e.g., Moldwin et al., 1995]. Many of the studies have generally focused on the dynamics and features of the plasmapause [e.g., Elphic et al., 1996; Moldwin et al., 2003, 2004] and the outer plasmasphere [e.g., Moldwin et al., 1995; Ganguli et al., 2000]. Moldwin et al. [1995], using the Los

\footnotetext{
${ }^{1}$ Atmospheric, Oceanic and Space Sciences, University of Michigan, Ann Arbor, Michigan, USA.

${ }^{2}$ Physics Department, University of Zambia, Lusaka, Zambia.

${ }^{3}$ Jet Propulsion Laboratory, California Institute of Technology, Pasadena, California, USA.

${ }^{4}$ Lunar and Planetary Laboratory, University of Arizona, Tucson, Arizona, USA.

Corresponding author: P. Sibanda, Atmospheric, Oceanic and Space Sciences, University of Michigan, Ann Arbor, MI 48109, USA. (sibandapatrick.ps@gmail.com)

C2012. American Geophysical Union. All Rights Reserved. 0148-0227/12/2012JA017522
}

Alamos (LANL) geosynchronous magnetospheric plasma analyzer (MPA) data, found that for moderate to high levels of geomagnetic activity the dusk sector of the outer plasmasphere has a fine-scale density structure. In another study, Moldwin et al. [2004] used the Combined Release and Radiation Effects Satellite (CRRES) plasma wave receiver measurements to characterize the plasmasphere-like features observed outside the plasmapause. In spite of these and many other studies on the structure and dynamics of the plasmasphere [e.g., Ganguli et al., 2000; Carpenter et al., 2002; Goldstein et al., 2003; Sandel et al., 2003; Goldstein, 2004; Darrouzet et al., 2008], some broad gaps in our knowledge of the plasmasphere still remain. One such gap is with regards to the azimuthal density structure and dynamics internal to the plasmasphere. Carpenter et al. [2000] observed and discussed deep density troughs inside the plasmasphere which appear in the aftermath of the plasmasphere erosion episodes and found this to be associated with the near-equatorial manifestations of geomagnetic field-aligned cavities. LeDocq et al. [1994] used the CRRES satellite data and obtained instances when the satellite encountered large quasiperiodic fluctuations in the electron number density inside the plasmasphere. Several other studies have examined the origins of the small-scale variability in the plasmasphere (both the outer and inner plasmasphere) [e.g., Fok et al., 1995; Adrian, 2004; Liemohn, 2004; Goldstein et al., 2005; Liemohn et al., 2006].

[3] Many other studies have analyzed the Extreme Ultraviolet (EUV) data from the IMAGE satellite and have identified a variety of plasmaspheric density structures of 
(a)

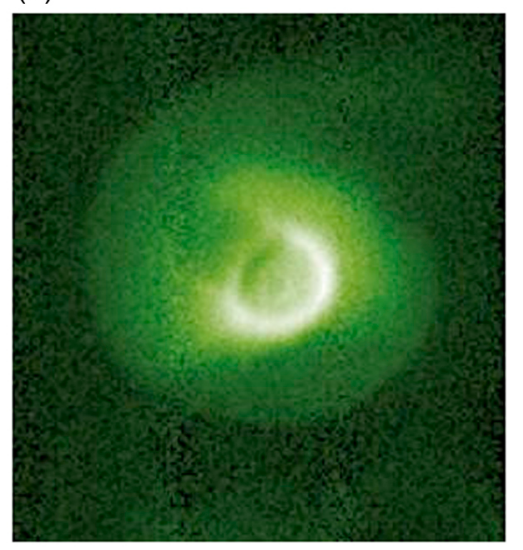

(b)

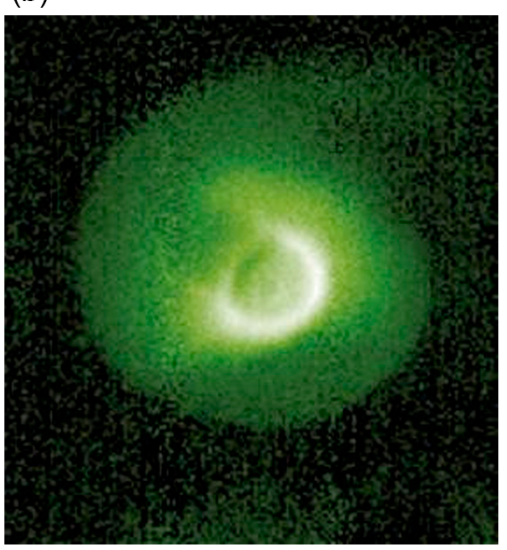

Figure 1. An original EUV image and its background-subtracted version. The images show sharp differences illustrating the usefulness of background subtraction. (a) Original image and (b) background subtracted.

varying scale sizes [e.g., Sandel et al., 2003; Goldstein, 2004; Gallagher et al., 2005; Pierrard and Cabrera, 2005]. For example, the plasmapause is characterized by a sharp drop in plasma density during geomagnetically disturbed conditions [Goldstein et al., 2003; Gallagher et al., 2005]. A significant plasmaspheric density structure is the plume formed due to enhancement of the global cross-tail electric field caused by magnetospheric convection [Nishida, 1966; Goldstein, 2004]. Other notable density structures include notches, plasmaspheric channels [Sandel et al., 2003], crenulations and shoulders [Darrouzet et al., 2008]. Notches have been observed to extend radially inward to less than $\mathrm{L}=2$ in some circumstances [Gallagher et al., 2005]. These structures are most distinguishable at the plasmapause and their connection to the inner plasmasphere are not fully understood.

[4] The inner plasmasphere exhibits a variety of other density enhancements that are only partially described and more work remains with regard to quantifying their scale sizes. The purpose of this work is to quantify the scale sizes of the azimuthal density structure of the plasmasphere internal to the plasmapause as seen by the Extreme Ultraviolet (EUV) imager on the IMAGE satellite. The study also also provides a better understanding of the origin of the density flactuations and how the observed features evolve with respect to enhanced geomagnetic activity.

\section{Methodology}

[5] The IMAGE satellite operated from 2000 to 2005 and the IMAGE EUV instrument provided global images of the plasmasphere and has been used in various studies to characterize the dynamics and features of the plasmasphere [Sandel et al., 2001]. Figure 1a shows an illustrative observation of the plasmasphere as viewed by the EUV instrument on the IMAGE satellite and shows a variety of plasmaspheric structures showing the Earth at the center, ionospheric airglow (bright arch) on the dayside and the shadow on the nightside. Photon counts increase logarithmically from dark green to light green and then white.

[6] The IMAGE spacecraft is in an elliptical polar orbit and the EUV instrument was designed to provide global snapshots of the entire plasmasphere when the spacecraft is at apogee. The EUV instrument consists of three cameras sensitive to 30.4-nm extreme ultraviolet light that is resonantly scattered by the $\mathrm{He}^{+}$ions in the plasmasphere. Assuming an optically thin plasmasphere, the column-integrated brightness of scattered EUV photons as viewed from the spacecraft is directly proportional to the density of $\mathrm{He}^{+}$ions integrated along the EUV instrument's line of sight [Ganguli et al., 2000; Galvan et al., 2008]. Thus brightness changes in an EUV image generally correspond to density variations in the plasmasphere [e.g., Ganguli et al., 2000; Sandel et al., 2003].

\subsection{Data Processing}

[7] The processing procedure included applying a background subtraction routine on each EUV image before azimuthal brightness values were extracted. This was done to reduce the uncertainties in the mean pixel brightness values caused by changes in the scattered sunlight contamination (illustrated in Figure 1). This therefore ensures that the difference in brightness between different images considered is mostly due to density variations in the plasmasphere [Galvan et al., 2008]. The background subtraction serves to increase the signal-to-noise ratio of the azimuthal profile.

[8] Figures 1a shows an original EUV image taken at 18:45 UT on 02 June 2001 and Figure $1 \mathrm{~b}$ shows the same image after background subtraction. The sharp contrast between the images shows the importance of doing background subtraction. The background-subtracted images were corrected for perspective effects by mapping points in the image to the geomagnetic equatorial plane along magnetic field lines using dipole geomagnetic field geometry [Roelof and Skinner, 2000; Sandel et al., 2003]. This procedure repositions the brightness value of each pixel and the result is a projected image shown in Figure 2a for the same image in Figure 1. In addition, the mapping routine also identifies which pixels lay on the areas of seam overlap between the three EUV sensors and the Earth's shadow producing a shadow-mask image shown on Figure 2b.

[9] The shadow-mask image is useful for determining which pixels in the resultant image are valid and potentially reliable for photometry. In the areas of seam overlap between 
(a)

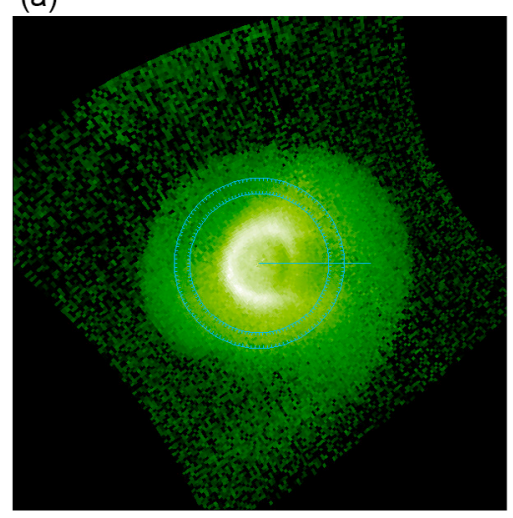

(b)

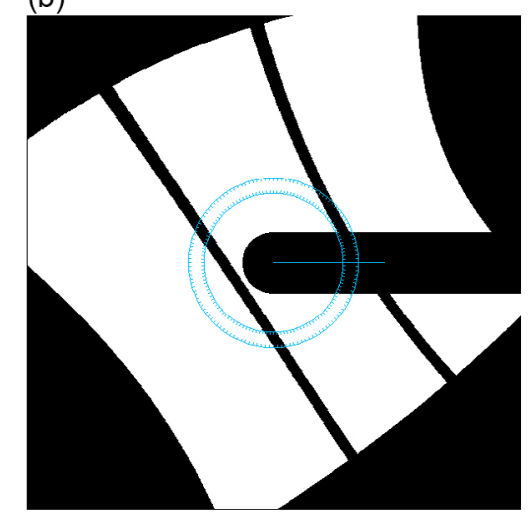

Figure 2. (a) Representation of the final image mapped to the geomagnetic equatorial plane. (b) Representation of the shadow-mask image showing location of the seams between the three EUV sensors (the black narrow bars) and the dark bar on the left represents Earth's shadow. The blue circles represent the annulus from which mean pixel brightness values were extracted.

the three EUV sensors, brightness values are unreliable as structure arises from incompletely corrected vignetting at the edges of the camera fields, while values in the shadow region are lower because of reduced illuminations. In either case, these regions are not useful for photometry.

[10] The blue circles in Figure 2 represent the annulus of thickness $\mathrm{L} \approx 0.5$ and centered at a radius of $\mathrm{L}=2.5$ from which the brightness profiles were extracted. The annulus was divided into 128 bins separated by $\approx 11.25 \mathrm{~min}$ in magnetic local time (MLT). The brightness values for each bin were calculated by averaging the brightness values of the pixels in each bin. The typical number of pixels in the bin ranged between 75 and 95 . As a measure of the uncertainty of the mean brightness for each bin, the standard deviation $\left(\sigma_{1}\right)$ of the mean for the brightness values of pixels in the given bin was used. The study is focused on the inner plasmasphere, therefore the range in L-shell of the annulus was chosen so that the brightness values are taken from a region of relatively uniform volume of plasmaspheric plasma free from the features of the plasmapause and trough at L-shells $\gtrsim 3$ and free from the effects of airglow at L-shells $\lesssim 2$.

\subsection{Data Analysis}

[11] The mean brightness profiles taken from an annulus at $\mathrm{L}=2.5$ of the EUV images allow us to characterize the azimuthal structure of the inner plasmasphere. This allows us to quantify the spatial scales in magnetic local time of the observed density structures. The analysis also enables a quantitative study of the relative amplitude of the density depletions/enhancements with respect to the adjacent walls of the identified plasmaspheric density structures as well as to characterize typical scale sizes of the azimuthal structure and how they evolve with respect to geomagnetic activity. Analysis involved fitting a high-order polynomial, whose order was chosen to fit the diurnal trend [e.g., Galvan et al., 2008] (described in the section that follows).

\section{Results}

[12] Figure $3 \mathrm{~b}$ displays a representation of the azimuthal brightness profile extracted from the image shown in
Figure 3a. Note the diurnal variation of the mean brightness (photon count) which shows a slight decrease in the day time sector followed by a large increase to a maximum in the noon sector and then drops to a minimum in the nighttime. Fitting a high-order polynomial and subsequently subtracting it from the original data shows that there are considerable brightness fluctuations within the azimuthal structure of the plasmasphere. This is evident in the plot of the residuals shown at the bottom of Figure 3b. The error bars shown on the original data curve (red) represent the standard deviation of the mean brightness value in each bin $\left(\sigma_{1}\right)$. This provides an estimate of the noise level in the brightness profiles and enables us to ensure the identified density structures represent actual geophysical signatures and not noise and artifacts related to the viewing geometry, the camera seams and the Earth's shadow. Brightness fluctuations with amplitudes that fall below the standard deviation of the mean represented by the error bars are not considered significant.

\subsection{Density Structures}

[13] The plots of the residuals in Figures $3 b, 4 a, 4 b$ and $4 c$ reveal brightness fluctuations of a wide range of amplitudes within the single UT snapshot of a 24 hour of MLT azimuthal profiles of the Earth's plasmasphere. This suggests that there is density structure of varying amplitudes in the inner plasmasphere. Some density structures such as notches are clear and distinct and can be easily identified from the EUV images e.g., the notch labeled in Figure 3a. The drop in brightness observed between 12:00 and 15:00 MLT in Figure $3 \mathrm{~b}$ corresponds to this notch. The plot reflects a relative drop in photon count of $\sim 30(50 \%)$ with respect to the adjacent walls associated with the notch and the width in MLT is $\sim 3$ hours. This represents an example of a broad notch that extended deep into the inner regions of the plasmasphere to less than $\mathrm{L}=2.5$ [Gallagher et al., 2005]. Figure $3 \mathrm{c}$ shows the same notch approximately 6 hours later, and the corresponding brightness profile shown in Figure 4c shows that, in addition to shifting to the dusk sector as a result of corotation, the notch was depleted further to $\sim 40$ $(\sim 70 \%)$ photon counts below the adjacent walls representing a further drop by a factor of $1 / 3$. Examining Figure $3 a$ in 

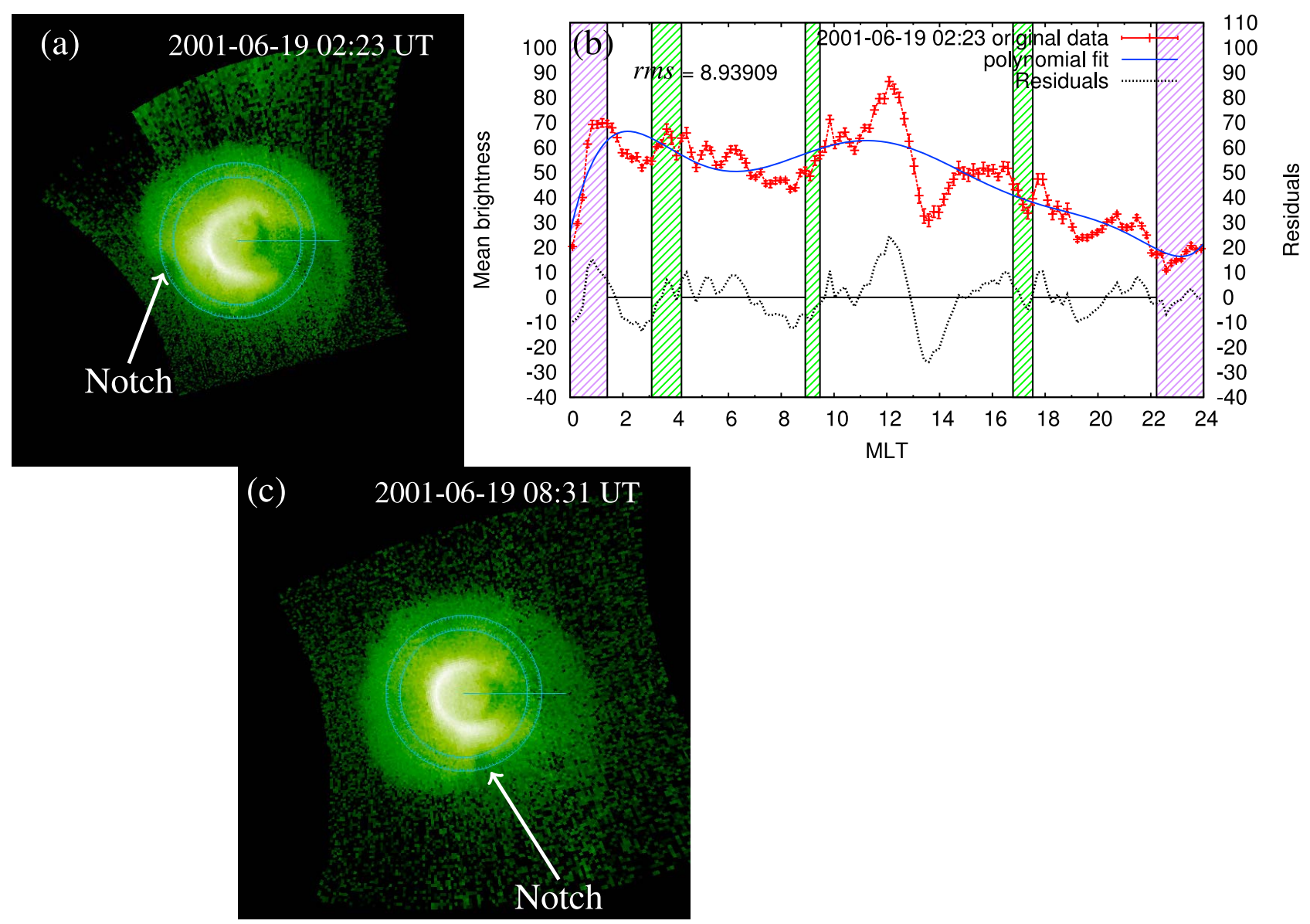

Figure 3. (a) The EUV image (taken on day 19 June 2001 at 02:23 UT) mapped to the geomagnetic equatorial plane. The blue circles represent the annulus from which the (b) mean brightness profile was extracted. Shown on the brightness profile are the seam regions and the Earth's shadow region. The error bars indicate the variability of the pixel to pixel brightness within each bin. The plot of the residuals at the bottom of Figure $3 \mathrm{~b}$ were calculated by fitting a 7th order polynomial to the profile. (c) The EUV image taken 6 hours after the image in Figure 3a showing the notch shifted to the dusk sector within the 6 hour period.

comparison with Figure 3c taken 6 hours apart shows that the plasmapause expanded outward either due to refilling or outward radial motion or other potential mechanisms that are discussed in more detail in the discussion section.

[14] The brightness profiles also reveal other significantly large morphological features characterized by density enhancements and rapid density fluctuations that are not easily noticeable by visual inspection of the original EUV image shown in Figure 3a. For example the brightness profile (Figure $3 \mathrm{~b}$ ) extracted from the image shows considerable brightness enhancements $(>25 \%)$ observed between 01:00 and 02:00 MLT and also between 11:00 and 13:00 MLT. The brightness profile also shows some fairly large rapid fluctuations (with amplitude enhancements of about 10-30\% and periods of around half an hour) between 03:00 and 06:00 MLT.

[15] The same structures are observed on the images taken at subsequent UT times shown on Figure 4, however, they are shifted to different magnetic local time sectors as a consequence of the plasma corotating with the Earth. For example the brightness enhancement feature observed at the 01:00-02:00 magnetic local time sector (at the edge of the shadow region) on Figure $3 \mathrm{~b}$ moved to the 04:00-06:00 magnetic local time sector on Figure 4c which was taken approximately 6 hours later. Further investigation of the profiles (Figures 3b, 4a, 4b and 4c) shows about four regularly spaced peaks over the entire azimuthal length. This suggests some quasiperiodic azimuthal structure of approximately 6 hours MLT within the plasmasphere. [Adrian, 2004] also reported similar apparent periodic azimuthal structures in the plasmasphere when they analyzed the bifurcated radial enhancements in the $\mathrm{He}^{+}$distribution observed by IMAGE EUV. In addition to the structures with fairly large amplitude undulations, the profiles also show some rapid brightness fluctuations of relatively smaller amplitudes and length-scales. The observed fluctuations indicate that the inner plasmasphere exhibits both fine-scales (order of $10 \mathrm{~s}$ of minutes) and meso-scale (order of a few hours) structure.

[16] Investigation of the geomagnetic activity conditions during the period when these images were taken, shows that a relatively strong geomagnetic storm (with maximum $K p \sim 5$ ) occurred between June 17 and 20, 2001 shown by the plot of the $K p$ index on Figure 5. The images where 

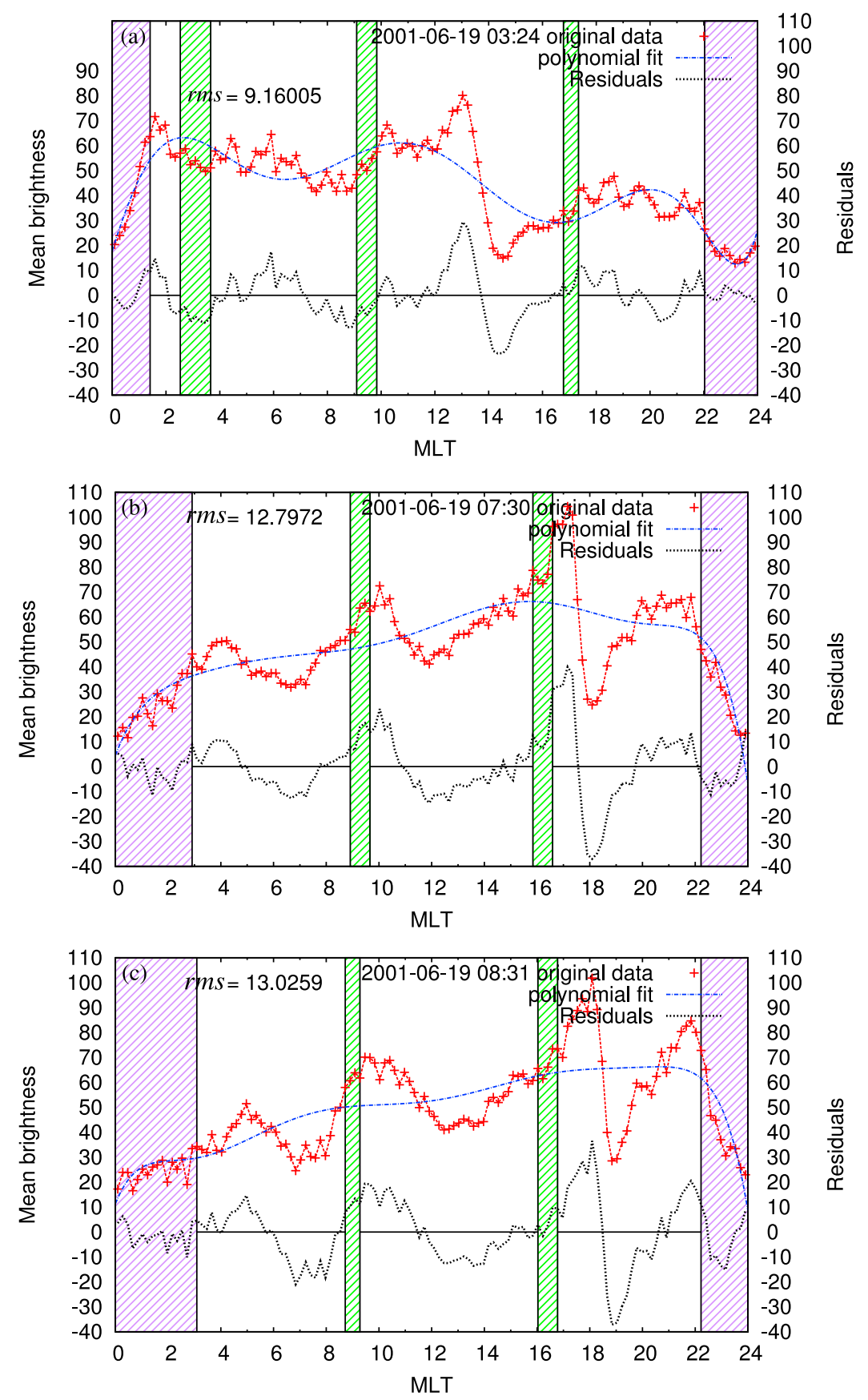

Figure 4. Brightness profiles, for 3 images taken at different UT times after the image shown on Figure 3a. The profiles show the density enhancement features identified in Figure $3 \mathrm{~b}$ present, however corotated to different local time sectors.

taken in the aftermath of this storm. To investigate the origins of the observed structures, the images were analyzed in comparison with those taken before and during the storm period. Figure 5 shows a plot of the $K p$ index for the June 17-20 storm and the labels (a, b, c and d) indicating the respective moments when the brightness profiles shown in Figure 6 were taken. For this storm, the images taken during the main phase of the storm were not usable and are not included in the analysis.

[17] Figure 6 shows the brightness profiles for four images: two (Figures $6 \mathrm{a}$ and $6 \mathrm{~b}$ ) taken at the commencement and the other two (Figures 6c and 6d) taken in the aftermath of the storm. Analysis of the images taken at the different stages of the storm activity shows that the density structures appear enhanced and the quasiperiodic behavior is more pronounced in the images taken in the aftermath of the geomagnetic storm. For the two profiles taken prior to the storm, the fluctuation amplitudes are relatively smaller and the quasiperiodic structure is generally absent.

[18] Four other geomagnetic storm periods (shown in Figure 7) that had a maximum $K p$ of at least 5 and had EUV images that were usable were identified. For all the storms, 


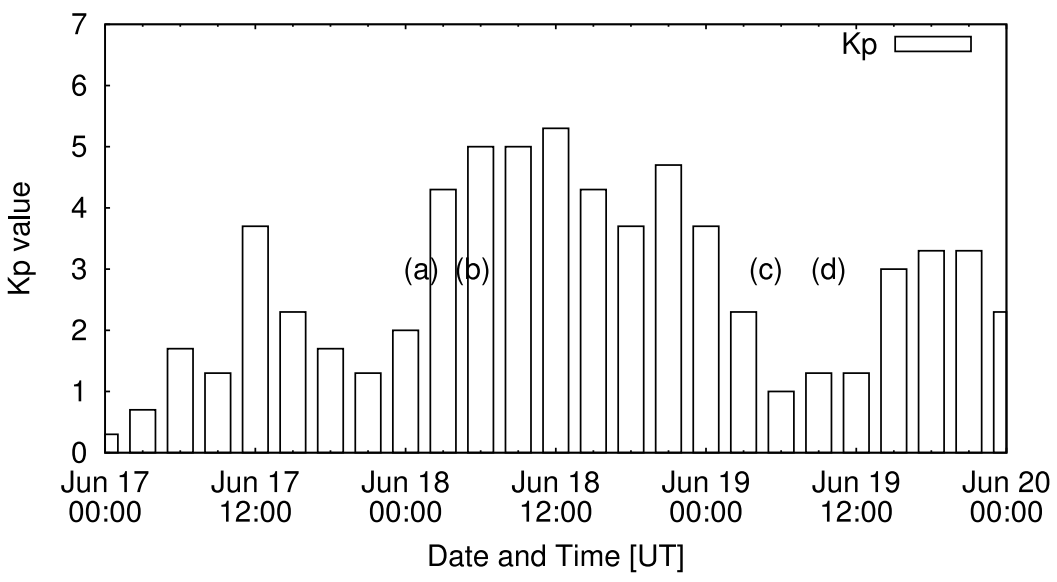

Figure 5. $K p$ index plot for the period 17-20 June 2001. The labels a-d indicate moments when the selected IMAGE EUV images shown on the corresponding plots in Figure 6 were taken.

the same trend was observed with the images taken after the storm having enhanced rapid density fluctuation amplitudes forming a quasiperiodic structure while those taken prior to the storm had lower fluctuation amplitudes and the periodic structure generally missing. The observations here are consistent with interpretation of Chappell [1974], Carpenter et al. [1979] and Goldstein et al. [2003] that much of this fine-scale structure originates from the interplay among global scale magnetospheric convection and electric fields associated with the Earth's rotation. Moldwin et al. [1995], determined that the presence of fine-scale density structure in the outer plasmasphere is correlated with substorms and is possibly caused by the penetrating electric fields associated with a substorm. These observations suggest that the finescale and meso-scale density structure internal to the plasmapause are driven by storm dynamics. These observations demonstrate the complexity of the azimuthal plasmaspheric behavior, not only at the plasmapause, but inside the main plasmasphere as well.

\subsubsection{Scale Sizes and Amplitudes}

[19] The length scales of the observed meso-scale density structures are on the order of $\sim 2-3$ hours of MLT and the relative amplitudes with respect to the adjacent walls range between $\sim 10-60$ photon counts representing relative enhancements or depletions $\left(\frac{\Delta n}{n}\right)$ by factors of $\sim 2-5$. The fine-scale rapid amplitude fluctuations are on the order of
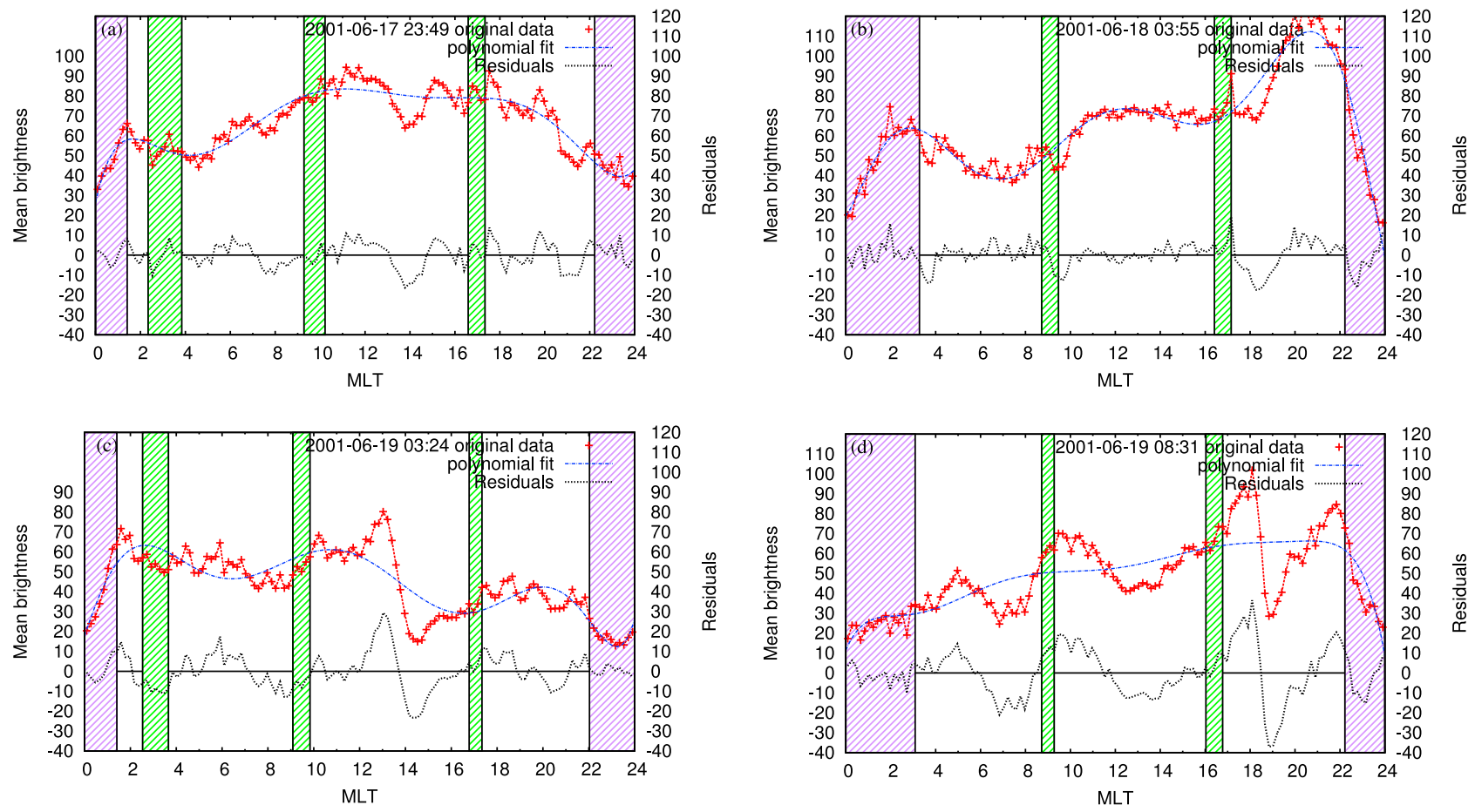

Figure 6. Brightness profiles for the EUV images taken at the times indicated on Figure 5. (a and b) The profiles correspond to images taken at the commencement of the storm. (c and d) The profiles correspond to images taken in the aftermath of the storm. 
(a)
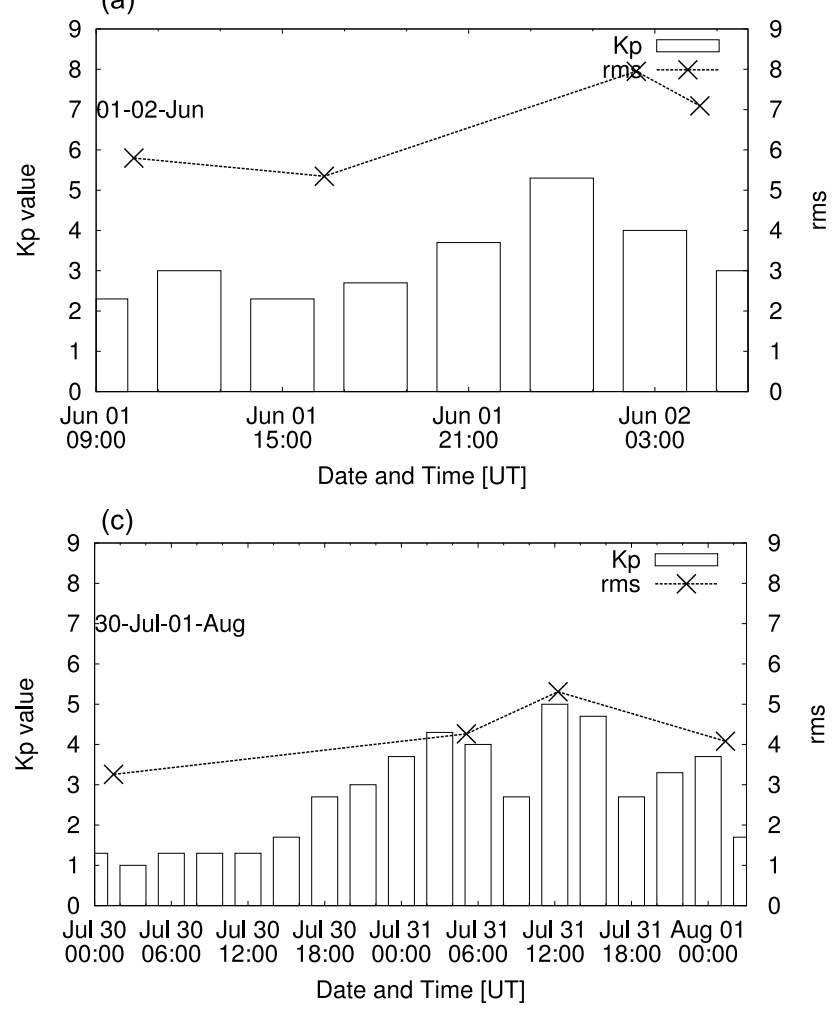

(b)
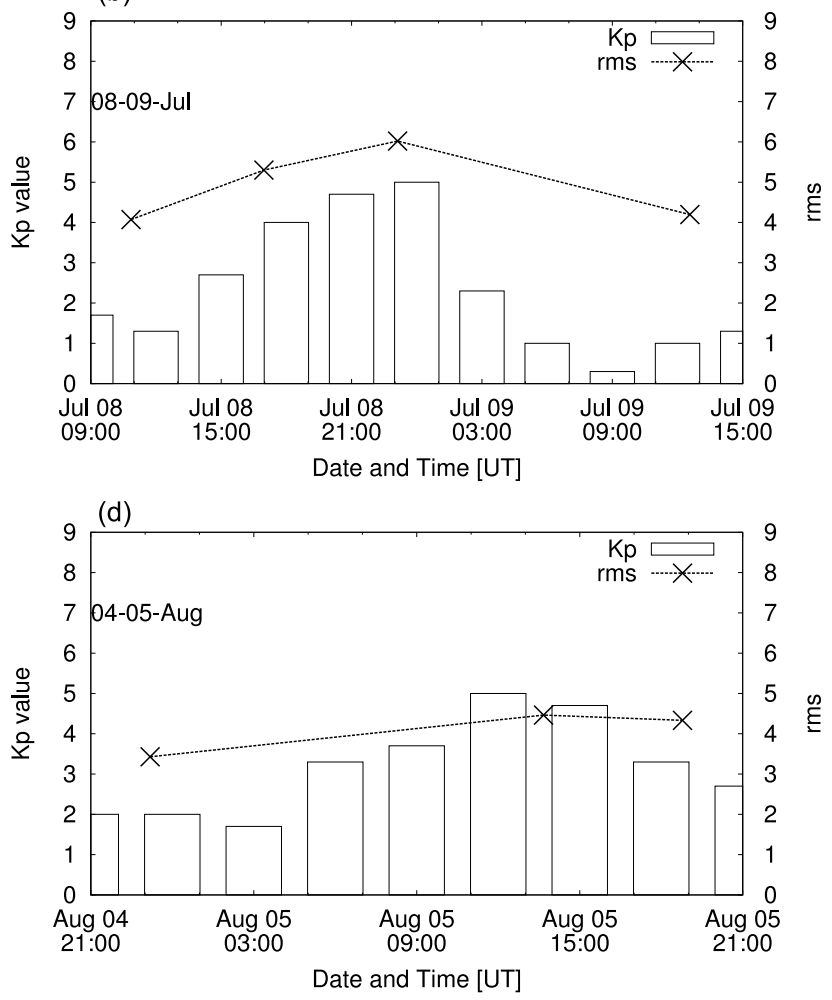

Figure 7. Plots showing the relationship between the $K p$ index and the level of variability indicated by the rms during four geomagnetic activity events.

10 s of minutes to an hour. Owing to their relatively small scale sizes, these density structures are not easy to identify by visual inspection of the original EUV images. Analysis of the EUV images by extracting the brightness values, therefore enables us to identify more density structures within the inner plasmasphere and also to estimate their scale sizes.

\subsubsection{Variability}

[20] Analysis of the brightness profiles has revealed significant meso-scale and fine-scale density fluctuations within the azimuthal plasmaspheric structure. As observed, the scale sizes of the fluctuations vary significantly from image to image. To investigate this variability, the root mean square of the residuals ( $r m s$ ) shown on each plot was calculated from the fitted polynomial and is used to quantify the level of variability for each profile. The rms indicates an estimate of the deviations of the data around the fitted polynomial and provides a measure of the small scale density variations about the mean (the polynomial fit). A large value of the rms indicates increased variability. Figure 7 displays four geomagnetic storm events of similar rise in the $K p$ index value with maximum $K p \sim 5$. Each plot shows the rms calculated for images taken at different stages of each geomagnetic storm activity plotted together with the $K p$ index. The $K p$ index provides the measure of geomagnetic activity. It is clear from the plots that the fluctuations are greater during the disturbed periods as shown by the greater value of the rms for a high $K p$ value. This represents a relative increase in variability of about $13 \%$ corresponding to a rise in $K p$ of about 2 to 3 . This shows how the level of variability changes as a function of geomagnetic activity showing a general trend of increasing variability with increasing $K p$.

\section{Discussion}

[21] We have analyzed the azimuthal brightness profiles extracted from successive snapshot images during selected IMAGE passes for 5 storm intervals to characterize the azimuthal structure and dynamics of the inner plasmasphere. The results revealed that the inner plasmasphere exhibits both meso-scale (the large 'density enhancement and/or drop features' with scale sizes of 2-3 hours in MLT and amplitudes of 10-40 photon counts above the adjancent walls) and fine-scale ('rapid density fluctuations' with scale sizes of $10 \mathrm{~s}$ of minutes to an hour and amplitudes of less than 10 photon counts above the adjancent walls) structure. The meso-scale features observed in Figure $3 \mathrm{~b}$ can also be tracked in the subsequent images (Figures $4 \mathrm{a}, 4 \mathrm{~b}$ and $4 \mathrm{c}$ ). This suggests that the features represent actual density structures and not just noise related to the Earth's shadow or the camera seams. The rapid density fluctuations suggest that, like the outer plasmasphere as observed by Moldwin et al. [1995], the inner plasmasphere also exhibits significant fine-scale density structure. Another observation worth noting is that the azimuthal structure shows considerable undulation behavior. The 'density enhancement structures' appear to occur at quasiregular intervals of approximately 6 hours of MLT in the aftermath of enhanced geomagnetic activity, an observation 
that indicates a systematic azimuthal distribution of density in the inner plasmasphere. We have demonstrated this for five events, and all the events consistently have the same behavior.

[22] Comparison of many successive snapshot images during a selected IMAGE pass demonstrate how the density structures corotate with the Earth [e.g., Gallagher et al., 2005; Sandel et al., 2003; Burch et al., 2004; Galvan et al., 2010]. This study has also provided more insight into the dynamics of the inner plasmasphere showing how as the plasmapause moved outward due to refilling and radial motion following a plasmaspheric erosion episode, the notch initially seen at the plasmapause is seen deeper inside the plasmasphere suggesting that some structure internal to the plasmasphere is from "fossil" plasmapause features. The observed plasmasphere's outward expansion could also be attributed to a redistribution of plasmaspheric plasma happening through the corotation of the plasmasphere onto the elongated drift paths as the bulge in the dusk sector rotates toward the morning sector [Carpenter, 1967; Horwitz et al., 1981]. However, in this case we observe a more localized expansion restricted to the region outside the dusk sector. Such expansion is presumed to occur through the filling of flux tubes that were depleted during the previous geomagnetically disturbed period [Horwitz et al., 1981]. The "notch" remains observable in the inner plasmasphere while the plasmapause expands outwards to larger L. This indicates that the meso-scale density feature is somewhat disconnected from the new plasmapause dynamics and hence we refer to it as a "fossil" feature.

[23] Computing the rms of the residuals and using this to provide a measure of the level of variability in each image and determining how the variability evolves with respect to convection enhancements shows a trend where increase in magnetic activity corresponds to increased variability. Images taken during the most disturbed activity periods have higher density variability within the plasmaspheric azimuthal structure. This suggests that the increase in variability may be imposed by changes in the large-scale convection electric fields during geomagnetic storms. A high value of $K p(>3)$ indicates enhanced geomagnetic activity which essentially corresponds to increased magnetospheric convection [Goldstein et al., 2003] strong enough to cause motion of the plasmasphere and cause erosion of the plasmapause [see also Siscoe, 1966; Hughes, 1988]. This observation is in agreement with the findings by Goldstein et al. [2003] that rapid fluctuations in convection strength lead to azimuthal structure being created by inhomogeneities in the global convection field and by finite penetration of this convection field to inside of the plasmasphere. The results presented in Figure 7 suggest that the observed relative increases in variability are caused by convection enhancements. Other processes such as the ring current also can influence the plasmasphere structure and dynamics through the injection of a high-energy plasma component into the plasmasphere [Ganguli et al., 2000]. The processes by which the ring current can interact with the plasmasphere include Coulomb collisions [see Fok et al., 1995], charge exchange and wave-particle interactions [Hughes, 1988; Ganguli et al., 2000]. Waves affect the macroscopic plasma state by influencing the transport properties and therefore do play an important role in the overall dynamics of space plasma.

\section{Summary and Conclusions}

[24] Much can be learned about the azimuthal structure of the inner plasmasphere from the IMAGE EUV data by analyzing brightness profiles extracted from a region inside the plasmasphere. The inner plasmasphere exhibits density features of varying scale sizes characterized by density enhancements and rapid density fluctuations.

[25] This study found that the inner plasmasphere displays significant fine-scale and meso-scale azimuthal density structure. The level of variability is strongly related with the convection enhancements as indicated by the $K p$ index with the variability considerably higher during geomagnetically disturbed periods. The increase in the level of variability is likely associated with rapid fluctuations in convection strength that are a result of the combination of inhomogeneities in the global convection field and the finite penetration of this convection field to inside of the plasmasphere. The observed structure may also be attributed to enhanced storm-time outflow, such as from subauroral infrared arcs, where localized heating could extend the ionospheric scaleheight and outflow to produce a localized enhanced plasmaspheric density.

[26] The interpretation of the observations presented here are consistent with observations made in previous studies of the features of the plasmasphere such as the suggestions by Goldstein et al. [2003] that rapid fluctuations in convection strength may lead to azimuthal structure being created in the inner plasmasphere. In addition, observations presented here suggest meso-scale azimuthal structure observed inside the inner plasmasphere are "fossil" remnants of plasmapause azimuthal structure suggesting sharp gradients can last for 10 s of hours deep inside the plasmasphere.

[27] Acknowledgments. This work was done while Patrick Sibanda worked as a Post-doc under the guidance of Mark Moldwin at the University of Michigan and was supported by NASA grants NNX09AI62G, NNX10AC82G and NNH09ZDA001-LWSSTRT. The IDL EUV analysis tools developed by the University of Arizona are available at http://euv. lpl.arizona.edu/euv/index. Work at the University of Arizona was supported under NASA Grant NNX07AG46G from the Heliospheric Guest Investigator Program.

[28] Masaki Fujimoto thanks the reviewers for their assistance in evaluating this paper.

\section{References}

Adrian, M. L. (2004), IMAGE EUV observation of radially bifurcated plasmaspheric features: First observations of a possible standing ULF waveform in the inner magnetosphere, J. Geophys. Res., 109, A01203, doi:10.1029/2003JA009974.

Burch, J. L., J. Goldstein, and B. R. Sandel (2004), Cause of plasmasphere corotation lag, Geophys. Res. Lett., 31, L05802, doi:10.1029/ 2003GL019164.

Carpenter, D. L. (1967), Relations between the dawn minimum in the equatorial radius of the plasmapause and Dst, Kp, and local $\mathrm{K}$ at Byrd Station, J. Geophys. Res., 72(11), 2969-2971.

Carpenter, D. L., C. G. Park, and T. R. Miller (1979), A model of substorm electric fields in the plasmasphere based on whistler data, J. Geophys. Res., 84(A11), 6559-6563.

Carpenter, D. L., R. R. Anderson, W. Calvert, and M. B. Moldwin (2000), CRESS observations of density cavities inside the plasmasphere, J. Geophys. Res., 105(A10), 23,323-23,338.

Carpenter, D. L., M. A. Spasojevicć, T. F. Bell, U. S. Inan, B. W. Reinisch, I. A. Galkin, R. F. Benson, J. L. Green, S. F. Fung, and S. A. Boardsen 
(2002), Small-scale field-aligned plasmaspheric density structures inferred from the Radio Plasma Imager on IMAGE, J. Geophys. Res., 107(A9), 1258, doi:10.1029/2001JA009199.

Chappell, C. R. (1974), Detached plasma regions in the magnetosphere, J. Geophys. Res., 79(13), 1861-1870, doi:10.1029/JA079i013p01861.

Darrouzet, F., et al. (2008), Plasmaspheric density structures and dynamics: Properties observed by the CLUSTER and IMAGE missions, Space Sci. Rev., 145(1-2), 55-106, doi:10.1007/s11214-008-9438-9.

Elphic, R. C., L. A. Weiss, M. F. Thomsen, D. J. McComas, and M. B. Moldwin (1996), Evolution of plasmaspheric ions at geosynchronous orbit during times of high geomagnetic activity, Geophys. Res. Lett., 23(16), 2189-2192.

Fok, M. C., P. D. Craven, T. E. Moore, and P. G. Richards (1995), Ring current-plasmasphere coupling through Coulomb collisions, in Cross-Scale Coupling in Space Plasmas, Geophys. Monogr. Ser., vol. 93, edited by L. Horwitz, N. Singh, and L. Burch, pp. 161-171, AGU, Washington, D. C.

Gallagher, D. L., M. L. Adrian, and W. M. Liemohn (2005), Origin and evolution of deep plasmaspheric notches, J. Geophys. Res., 110, A09201, doi:10.1029/2004JA010906.

Galvan, D., M. B. Moldwin, and B. R. Sandel (2008), Diurnal variation in plasmaspheric $\mathrm{He}^{+}$inferred from extreme ultraviolet images, J. Geophys. Res., 113, A09216, doi:10.1029/2007JA013013.

Galvan, D., M. B. Moldwin, B. R. Sandel, and G. Crowley (2010), On the causes of plasmaspheric rotation variability: IMAGE EUV observations, J. Geophys. Res., 115, A01214, doi:10.1029/2009JA014321.

Ganguli, G., M. Reynolds, and M. Liemohn (2000), The plasmasphere and advances in plasmaspheric research, J. Atmos. Sol. Terr. Phys., 62(17-18), 1647-1657, doi:10.1016/S1364-6826(00)00117-6.

Goldstein, J. (2004), Simultaneous remote sensing and in situ observations of plasmaspheric drainage plumes, J. Geophys. Res., 109, A03202, doi:10.1029/2003JA010281

Goldstein, J., M. Spasojevic, P. H. Reiff, B. R. Sandel, and W. T. Forrester (2003), Identifying the plasmapause in IMAGE EUV data using IMAGE RPI in situ steep density gradients, J. Geophys. Res., 108(A4), 1147, doi:10.1029/2002JA009475.

Goldstein, J., J. L. Burch, B. R. Sandel, S. B. Mende, P. C. Brandt, and M. R. Hairston (2005), Coupled response of the inner magnetosphere and ionosphere on 17 April 2002, J. Geophys. Res., 110, A03205, doi:10.1029/2004JA010712.

Horwitz, J. L., C. R. Baugher, C. R. Chappell, E. G. Shelley, D. T. Young, and R. R. Anderson (1981), ISEE 1 observations of thermal plasma in the vicinity of the plasmasphere during periods of quieting magnetic activity, J. Geophys. Res., 86(A12), 9989-10,001.
Hughes, A. R. W. (1988), Interesting features of convection within the plasmasphere, J. Atmos. Sol. Terr. Phys., 50(1), 41-43.

LeDocq, M. J., A. D. Gurnett, and R. R. Anderson (1994), Electron number density fluctuations near the plasmapause observed by the CRRES spacecraft, J. Geophys. Res., 99(A12), 23,661-23,671, doi:10.1029/94JA02294.

Liemohn, M. W. (2004), Dependence of plasmaspheric morphology on the electric field description during the recovery phase of the 17 April 2002 magnetic storm, J. Geophys. Res., 109, A03209, doi:10.1029/2003JA010304.

Liemohn, M. W., A. J. Ridley, J. U. Kozyra, D. L. Gallagher, M. F. Thomsen, M. G. Henderson, M. H. Denton, P. C. Brandt, and J. Goldstein (2006), Analyzing electric field morphology through data-model comparisons of the Geospace Environment Modeling Inner Magnetosphere/Storm Assessment Challenge events, J. Geophys. Res., 111, A11S11, doi:10.1029/2006JA011700.

Moldwin, M. B., M. F. Thomsen, S. J. Bame, D. McComas, and G. D. Reeves (1995), The fine-scale structure of the outer plasmasphere, J. Geophys. Res., 100(A5), 8021-8029, doi:10.1029/94JA03342.

Moldwin, M. B., S. Mayerberger, H. K. Rassoul, T. Barnicki, and R. R. Anderson (2003), Plasmapause response to geomagnetic storms: CRRES results, J. Geophys. Res., 108(A11), 1399, doi:10.1029/2003JA010187.

Moldwin, M. B., J. Howard, J. Sanny, J. D. Bocchicchio, H. K. Rassoul, and R. R. Anderson (2004), Plasmaspheric plumes: CRRES observations of enhanced density beyond the plasmapause, J. Geophys. Res., 109, A05202, doi:10.1029/2003JA010320.

Nishida, A. (1966), Formation of plasmapause, or magnetospheric plasma knee, by the combined action of magnetospheric convection and plasma escape from the tail, J. Geophys. Res., 71(23), 5669-5679.

Pierrard, V., and J. Cabrera (2005), Comparisons between EUV/IMAGE observations and numerical simulations of the plasmapause formation, Ann. Geophys., 23(7), 2635-2646, doi:10.5194/angeo-23-2635-2005.

Roelof, E. C., and A. J. Skinner (2000), Extraction of ion distributions from magnetospheric ENA and EUV images, Space Sci. Rev., 91, 437-459.

Sandel, B. R., R. A. King, W. T. Forrester, D. L. Gallagher, A. L. Broadfoot, and C. C. Curtis (2001), Initial results from the IMAGE Extreme Ultraviolet Imager, Geophys. Res. Lett., 28(8), 1439-1442.

Sandel, B. R., J. Goldstein, D. L. Gallagher, and M. Spasojevic (2003), Extreme Ultraviolet Imager observations of the structure and dynamics of the plasmasphere, Space Sci. Rev., 109(1-4), 25-46, doi:10.1023/B: SPAC.0000007511.47727.5b.

Siscoe, G. L. (1966), A unified treatment of magnetospheric dynamics with applications to magnetic storms, Planet. Space Sci., 14, 947-967. 